\title{
Compatibilidade entre a Qualidade do Ar Interno e a Percepção do Usuário
}

\author{
Melyssa M. G. Silva ${ }^{2}$, Antônio E. Pereira ${ }^{2}$, Isabela F. França ${ }^{1}$, \\ Bruna M. O. S. Cordeiro ${ }^{1}$, Iwens. G. Sene Junior ${ }^{1}$, Renato F. Bulcão-Neto ${ }^{1}$ \\ ${ }^{1}$ Instituto de Informática - Universidade Federal de Goiás (UFG) \\ Caixa Postal 74001-970 - Goiânia - GO - Brasil \\ ${ }^{2}$ Escola de Engenharia Elétrica, Mecânica e de Computação - UFG \\ Caixa Postal 74605-010 - Goiânia - GO - Brasil \\ melyssamariana@discente.ufg.br, brunacordeirodinf.ufg.br, \\ iwens@ufg.br, rbulcao@ufg.br
}

\begin{abstract}
Air pollution has progressively increased in recent years, becoming the most significant environmental risk to human health. For this reason, there must be an assessment of indoor air quality (IAQ) through data obtained by sensors and people's perception of whom frequent the monitored place. This paper describes an IoT-based solution for IAQ monitoring and how we calculate the compatibility between the sensed and perceived air quality.
\end{abstract}

Resumo. A poluição do ar tem aumentado progressivamente nos últimos anos, tornando-se o risco ambiental mais significativo para a saúde humana. Por isso, deve haver uma avaliação da qualidade do ar interno (QAI) por meio de dados obtidos por sensores e da percepção dos frequentadores do ambiente monitorado. Este artigo descreve uma solução baseada em IoT para monitoramento de QAI e mostra como calculamos a compatibilidade entre a qualidade do ar detectada e percebida.

\section{Introdução}

Estudos realizados pela EPA (do inglês Environmental Protection Agency) classificaram a poluição do ar interno entre os cinco principais riscos ambientais para a saúde pública [EPA 2021]. A fim de informar a população sobre o nível da qualidade do ar, foi criado o Índice de Qualidade do Ar (IQA) que atribui números em uma escala entre 0 e 400, e é usado para ajudar a determinar quando o ar presente no ambiente passa a ser prejudicial à saúde humana [Nigam et al. 2015].

Em média, as pessoas passam cerca de $90 \%$ do tempo em ambientes fechados climatizados, e as concentrações de poluentes, dependendo das condições internas, podem ser superiores à dos ambientes externos. Diversas condições no ambiente implicam diretamente na qualidade do ar; em [Kraus et al. 2019] são apresentados fatores físicos que podem elevar os níveis das concentrações dos poluentes, como ventilação, iluminação, conforto térmico, ruído, biofilia, localização, layout do espaço físico e comodidades.

Devido à comum falta de cuidado com a qualidade do ar e fiscalização dos ambientes, surge a Síndrome do Edifício Doente (SED), situação em que ambientes fecha- 
dos, sem a devida renovação do ar, possuem altas concentrações de poluentes causadores de fadiga, falta de concentração, irritação nos olhos e nariz, garganta e pele secas [Teixeira et al. 2005]. A SED pode ocorrer principalmente em ambientes onde as condições não são adequadas às legislações vigentes, impactando na produtividade e concentração dos indivíduos.

Assim, a pesquisa bibliográfica realizada teve direcionamento para o âmbito psicológico, tendo como ponto de partida a pesquisa descrita em [Mohammed et al. 2020]. A mesma aborda a percepção psicossocial sobre a incidência de poeira no interior de prédios e casas, com questionários físicos e uma abrangente avaliação quantitativa. Destaca-se a análise de qui-quadrado de Pearson, utilizada para identificar a relação entre os fatores e o teste de significância [Pearson 1900].

No entanto, há evidências sobre discrepâncias entre a percepção do usuário e a real qualidade do ar justificada pelo tempo de exposição do usuário [Pagel et al. 2018]. Quanto mais a correlação está estabelecida nos extremos (baixa e alta), a avaliação do ambiente pode estar comprometida. Nesses extremos, os sensores podem estar descalibrados ou os usuários foram expostos a má qualidade do ar por muito tempo.

Este trabalho faz parte de um projeto de iniciação científica em andamento com o objetivo de investigar a correlação entre as qualidades de ar interno sentida no ambiente e percebida pelos frequentadores desse ambiente. Os objetivos específicos incluem a coleta e o processamento de dados ambientais coletados por meio de um sistema IoT (do inglês Internet of Things) e a análise de questionários.

O artigo está assim organizado: a Seção 2 descreve a solução IoT, o questionário e o método de análise das respostas obtidas; e a Seção 3 relata o estado atual da pesquisa.

\section{Monitoramento da qualidade do ar interno}

\subsection{Sistema AirPure}

A IoT descreve uma rede de objetos físicos incorporados a sensores, software e outras tecnologias visando conectar e trocar dados com outros dispositivos e sistemas via Internet [Ashton et al. 2009]. Desenvolveu-se uma solução IoT, denominada AirPure, composta por sensores que monitoram a qualidade do ar em ambientes internos. A Figura 1 apresenta a case impressa em 3D e os componentes utilizados no AirPure.

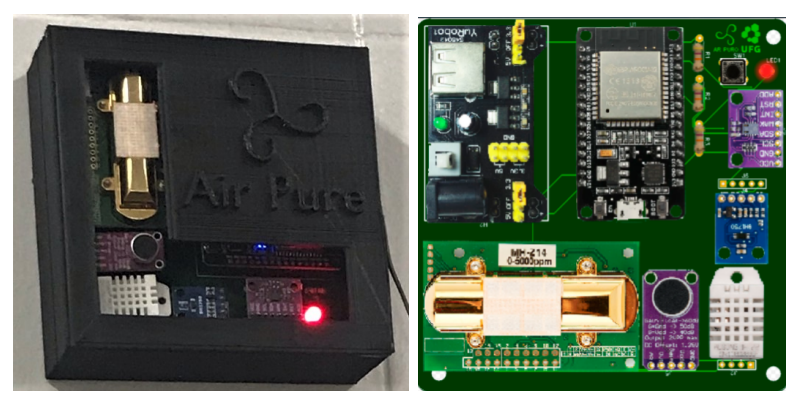

Figura 1. Protótipo AirPure.

A maioria dos dispositivos de análise da qualidade do ar, além de possuir valor elevado, faz a medição de parâmetros muito básicos, não alcançando uma análise completa. Baseando-se em legislações vigentes, foram conectados a um microcontrolador 
NodeMCU EsP21, sensores que englobam os parâmetros sugeridos pelas normativas expressos na Tabela 1. COVT e $\mathrm{CO}_{2}$ referem-se a compostos orgânicos voláteis totais e dióxido de carbono, respectivamente.

Tabela 1. Fatores Monitorados pelo AirPure.

\begin{tabular}{llccl}
\hline Sensor & Fator & Máximo & Mínimo & Legislação \\
\hline DHT22 & Umidade Relativa & $65 \%$ & $35 \%$ & Anvisa - No 09/2003 \\
DHT22 & Temperatura & $26^{\circ} \mathrm{C}$ & $20^{\circ} \mathrm{C}$ & Anvisa - No 09/2003 \\
MH-Z14A & $\mathrm{CO}_{2}$ & $1000 \mathrm{ppm}$ & $400 \mathrm{ppm}$ & Anvisa - N $09 / 2003$ \\
CCS811 & $\mathrm{COVT}_{\mathrm{O}}$ & $500 \mu \mathrm{g} / \mathrm{m}^{3}$ & - & Diretrizes da OMS \\
BH1750 & Iluminância & $1000 \mathrm{lux}$ & $500 \mathrm{lux}$ & NBR-5413 \\
MAX9814 & Ruído & $65 \mathrm{~dB}$ & $30 \mathrm{~dB}$ & NBR-1052 \\
\hline
\end{tabular}

A funcionalidade principal da programação embutida no dispositivo envolve configurar o wifi pelo celular, o transporte de dados via protocolo MQTT (Message Queuing Telemetry Transport), leituras e conversões periódicas dos sensores e salvar dados em um servidor Google Cloud Platform.

Disposto em duas partes, servidor e aplicação, o software trata os dados recebidos do dispositivo, salva-os no banco de dados e os apresenta graficamente em uma interface gráfica, como a da Figura 2. Na interface é possível visualizar dados do ambiente físico onde o dispositivo está instalado, o valores de IQA e de Índice de Correlação de Percepção (ICP) - descrito na subseção 2.2 - e os valores mais recentes lidos dos sensores. Uma escala de cores indica se os valores estão dentro do intervalo delimitado pelos valores máximo e mínimo contidos na Tabela 1: verde e vermelho simbolizam valores dentro e fora dos limites, e laranja, aqueles valores próximos dos limites.

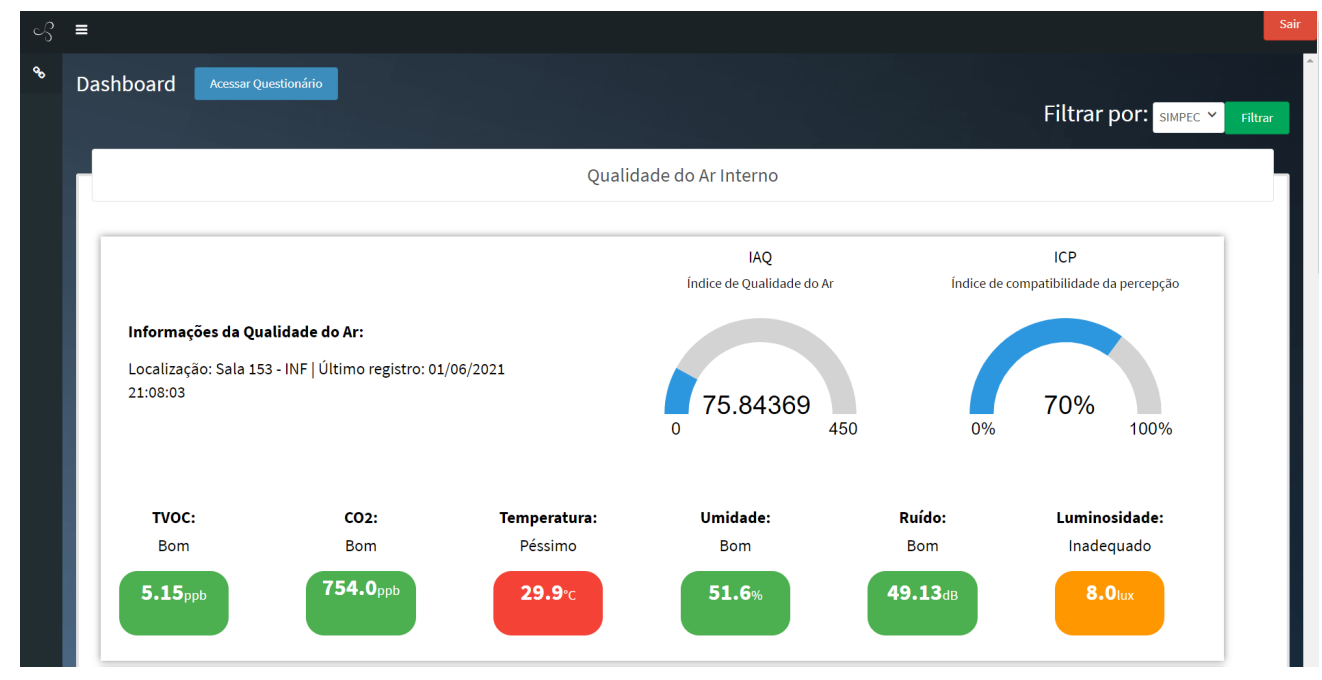

Figura 2. Interface do sistema de monitoramento de ambiente físico.

\section{2. Índice de Correlação de Percepção}

Baseado na regulamentação NBR-16401-3 [ABNT 2008], foram elaboradas perguntas de maioria subjetiva, na plataforma Google Forms. O questionário engloba faixa etária, localização, percepção, incidência de sintomas da SED, histórico de alergias e tabagismo. 
Aplicando a estatística de Pearson, o cálculo estabelece uma relação linear entre duas variáveis. Pearson estabelece uma relação que varia entre -1 e +1 , sendo 0 , sem correlação, e -1 e +1 os máximos níveis de correlação. Por exemplo, se o sensor mede 35 graus Celsius, de acordo com a norma vigente, caracteriza-se o ambiente como "Quente". Essa conceituação é comparada com a resposta do frequentador desse ambiente monitorado. Caso a pessoa informe que o ambiente está quente, então tais dados possuem um alto nível de correlação.

Dessa forma, o índice de correlação da percepção representa o quão relacionada está a sensação que as pessoas têm das condições de um ambiente com os dados reais coletados por sensores. Esse índice varia de 0 a $100 \%$ de compatibilidade, onde 0\% indica que não há nenhuma relação entre o que foi respondido e o valor coletado pelos sensores, e $100 \%$ indica que está totalmente relacionado.

\section{Estado atual das pesquisas}

Dois dispositivos AirPure estão instalados em um laboratório da Escola de Veterinária e Zootecnia da UFG, responsável pelo controle de qualidade de alimentos. Visa-se monitorar agentes ligados à qualidade do ar e a riscos ocupacionais, como o ruído. Resultados sobre a compatibilidade entre sensoreamento e percepção estarão disponíveis em breve.

\section{Referências}

ABNT (2008). Instalações de ar-condicionado - sistemas centrais e unitários parte 3: Qualidade do ar interior.

Ashton, K. et al. (2009). That 'internet of things' thing. RFID journal, 22(7):97-114.

EPA, U. S. E. P. A. (2021). Indoor air quality (iaq). Disponível em: https : / / www . epa.gov/indoor-air-quality-iaq/.

Kraus, M. et al. (2019). Assessment of the indoor environment for education. In IOP Conference Series: Earth and Environmental Science, volume 290, page 012144.

Mohammed, M. A. et al. (2020). Psychosocial perception of the effects of harmattan dust on the environment and health of building occupants in maiduguri, nigeria. Facilities (IF), 38(13/14):893-912.

Nigam, S. et al. (2015). Air quality index - a comparative study for assessing the status of air quality. Research Journal of Engineering and Technology, 6(2):267-274.

Pagel, E. C., Alvarez, C. E., and Reis Júnior, N. (2018). Avaliação e percepção do usuário em relação ao conforto térmico. Gestão e Tecnologia de Projetos, 13(3):57-77.

Pearson, K. (1900). On the criterion that a given system of deviations from the probable in the case of a correlated system of variables is such that it can be reasonably supposed to have arisen from random sampling. The London, Edinburgh, and Dublin Philosophical Magazine and Journal of Science, 50(302):157-175.

Teixeira, D. B. et al. (2005). Síndrome dos edifícios doentes em recintos com ventilação e climatização artificiais: Revisão de literatura. In VIII Congresso Brasileiro de Defesa do Meio Ambiente, pages 1-11, Rio de Janeiro, RJ, Brasil. 